\title{
Using Multi-Objective Evolutionary Algorithms in the Optimization of Polymer Injection Molding
}

\author{
Célio Fernandes ${ }^{1}$, António J. Pontes ${ }^{1}$, Júlio C. Viana ${ }^{1}$, and A. Gaspar-Cunha ${ }^{1}$
}

\begin{abstract}
A Multi-objective optimization methodology has been applied in the optimization of polymer injection molding process. This allowed the optimization of the operating conditions of the process from mold flow simulations, taking into consideration the existence of 5 criteria simultaneously, such as temperature difference on the molding at the end of filling, the maximum pressure, the pressure work, the volumetric shrinkage and the cycle time. The results produced shown that the proposed methodology is an efficient tool to be used in the optimization of this process.
\end{abstract}

\section{Introduction}

The injection molding process is one of the most important polymer processing technologies used to manufacture a great variety of plastics parts of high complexity and with tight dimensional tolerances. Injection molding of polymeric materials is a complex process involving several phases, such as plasticating (solids conveying, melting), melt flow (injection), pressurization (holding) and cooling/solidification. This, together with the viscoelastic nature of the polymeric materials, strongly affects the quality of the final molded parts.

The thermomechanical environment imposed to the polymer is determined by the operating conditions, the system geometry and the polymer properties. The final part morphology obtained, which determines their dimensions, dimensional stability and final properties, is controlled by the thermomechanical conditions defined by the process $[1,2]$. Therefore, due to the high number of variables involved and the strong interaction between them and the end product properties, the definition of the best operating conditions to use in a specific processing situation is a complex task. Sophisticated modeling programs, able to predict the process

${ }^{1}$ IPC- Institute for Polymer and Composites, Dept. of Polymer Engineering, University of Minho, Campus de Azurém, 4800-058 Guimarães, Portugal, \{cbpf, pontes, jcv, agc \}@dep.uminho.pt. 
response to the operating conditions defined, have been used for that purpose in an iterative way [3, 4]. The user defines some tentative processing conditions for the process under study and then these solutions are evaluated using the modeling routines in terms of the relevant criteria previously defined. If the desired response is not satisfactory the user defines new operating conditions, taking into account the results obtained for the previous ones, and the process continues until an acceptable performance is attained $[3,4]$.

However, this trial-and-error process is strongly dependent on the ability of the user to define the operating conditions to be tested. Thus, it is of great importance the application of an automatic optimization methodology able to define the operative window for the injection molding process without minimal user intervention.

Various optimization strategies using different methodologies to optimize the injection molding process have been reported in the literature [5-9]. Kim et al. [5] used Genetic Algorithms (GA) to optimize the processing variables (mold and melt temperatures and filling time) based on pre-defined criteria. The performance of the process was quantified using a weighted sum of the temperature difference, "overpack" and frictionally overheating criteria. Lotti and Bretas [6] applied Artificial Neural Networks, ANN, to predict the morphology and the mechanical properties of an injection molded part as a function of the processing conditions (mould and melt temperatures and flow rate). A central composite design of experiments approach was used to predict the molding morphology as a function of the processing conditions using the MoldFlow software. Castro et al. [7] used ANN and data envelopment analysis (i.e., statistical analysis) to find the optimal compromise between multiple performance measures to define the setting of injection molding variables and the gate location. Peic and Turng [8] used three different optimization algorithms (evolution strategies, differential evolution and simulated annealing) to optimize the injection molding processing conditions as a function of cycle time and volumetric shrinkage, using as restrictions the clamping force, the injection pressure and the temperature of the part at ejection. The relation between the processing conditions and the optimization criteria was performed with the CMOLD software. Finally, Alam et al. [9] applied a MultiObjective Evolutionary Algorithm (MOEA) to optimize the shrinkage of the molding and perform the runner balancing. Gaspar-Cunha and Viana [10] optimized the mechanical properties of injection molded parts using an MOEA approach.

In this work an optimization methodology based on MOEA is used for optimizing the operating conditions (melt and mold temperatures, injection flow rate, switchover point, holding time and pressure) in injection molding. This problem was not solved in the previous works above identified. A study about the performance of the proposed methodology using various optimization criteria has been carried out. The MOEA was linked with an injection molding simulator (CMOLD), which is able to compute the optimization criteria as a function of the 
defined operating conditions and able to take into account, simultaneously, the system geometry and polymer properties.

\section{Multi-Objective Evolutionary Algorithms}

The use of computer simulations on the design stages of engineering plastic components for the injection molding process is very frequent $[3,4]$. Initially, a finite element mesh representative of the part geometry is defined, the materials are selected, the gate location is defined and the initial processing variables are introduced. Then, after launching the simulation the outputs are analyzed. A trial-anderror process is applied, where the initial conditions, in what concerns geometry, material and/or processing conditions are modified until the desired results are obtained. This process can be very complex since in most cases multiple criteria are to be optimized simultaneously. Also, the finding of a global optimum solution is not guaranteed.

The methodology proposed in this work integrates the computer simulations of the injection molding process, an optimization methodology based on evolutionary algorithm and multi-objective criteria in order to establish the set of operative processing variables leading to a good molding process. The optimization methodology adopted is based on a Multi-Objective Evolutionary Algorithm (MOEA) [11], due to multi-objective nature of most real optimization problems, where the simultaneous optimization of various, often conflicting, criteria is to be accomplished [10-15]. The solution must then result from a compromise between the different criteria. Generally, this characteristic is taking into account using an approach based on the concept of Pareto frontiers (i.e., the set of points representing the trade-off between the criteria) together with an MOEA. This enabled the simultaneously accomplishment of the several solutions along the Pareto frontier, i.e., the set of non-dominated solutions $[14,15]$.

The link between the MOEA and the problem to solve is made in two different steps (see flowchart of Fig. 1). First, the population is randomly initialized, where each individual (or chromosome) is represented by the binary value of the set of all variables. Then, each individual is evaluated by calculating the values of the relevant criteria using the modeling routine (in this case CMOLD). Finally, the remaining steps of a MOEA are to be accomplished. To each individual is assigned a single value identifying its performance on the process (fitness). This fitness is calculated using a Multi-Objective approach as described in details elsewhere [12, 15]. If the convergence criterion is not satisfied (e.g., a pre-defined number of generations), the population is subjected to the operators of reproduction (i.e., the selection of the best individuals for crossover and/or mutation) and of crossover and mutation (i.e., the methods to obtain new individuals for the next generation). The RPSGAe uses a real representation of the variables, a simulated binary crossover, a polynomial mutation and a roulette wheel selection strategy $[11,14]$. 


\section{Injection Molding Optimization}

The optimization methodology proposed will be used for setting the processing conditions of the molding shown in Fig. 2, which will be molded in polystyrene (STYRON 678E). The relevant polymer properties used for the flow simulations were obtained from the software (CMOLD) database. A mesh with 408 triangular elements has been used. The simulations considered the mold filling and holding (post-filling) stages. A node near the P1 position, pressure sensor position (see Fig. 2) was selected as a reference point for this study. The processing variables to optimize and allowed to varied in the simulations in following intervals were: injection time, $t_{i n j} \in[0.5 ; 3] \mathrm{s}$ (corresponding to flow rates of 24 and $4 \mathrm{~cm}^{3} / \mathrm{s}$, respectively), melt temperature, $T_{i n j} \in[180 ; 280]{ }^{\circ} \mathrm{C}$, mold temperature, $T_{w} \in[30$; $70{ }^{\circ} \mathrm{C}$, holding pressure, $\mathrm{Ph} \in[7 ; 38] \%$ of maximum machine injection pressure, with fixed switch-over point, SF/P, at 99\%, holding time, t2P, of 30s and timer after hold pressure of $15 \mathrm{~s}$.

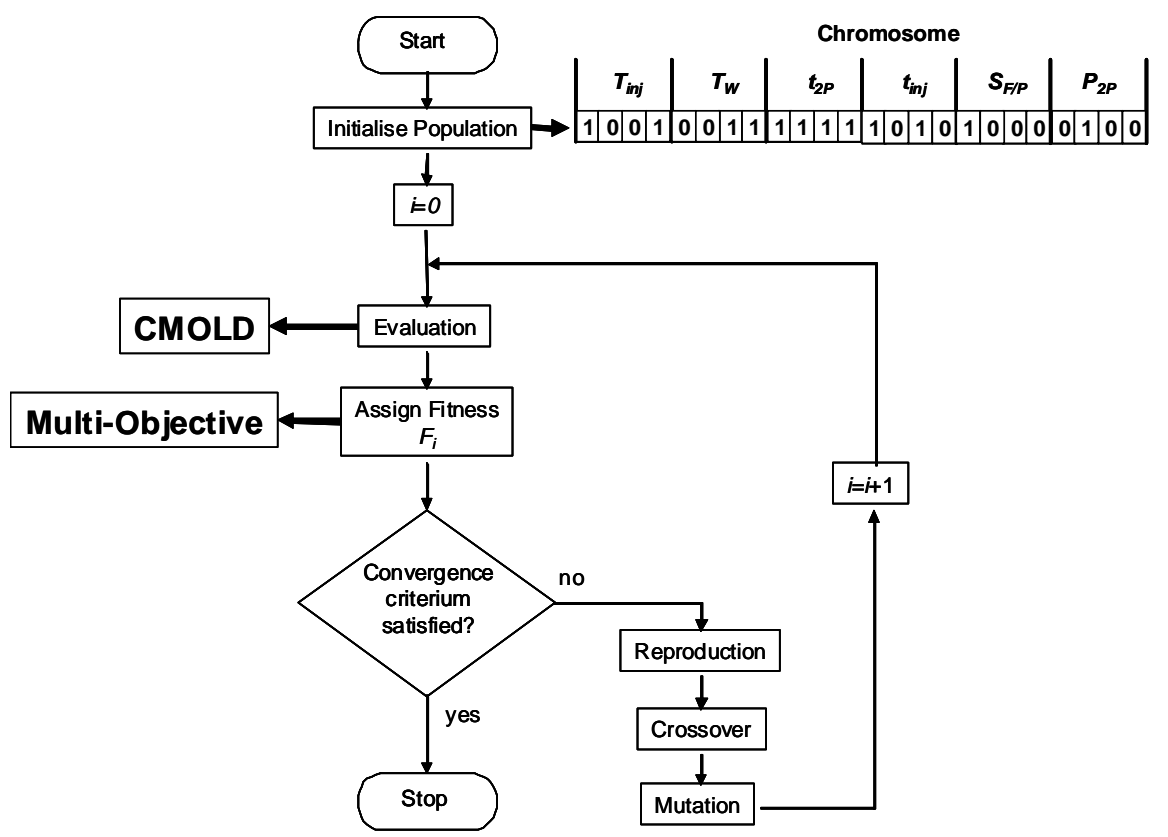

Fig. 1. Flowchart of the MOEA applied for the optimization of the injection molding process. 


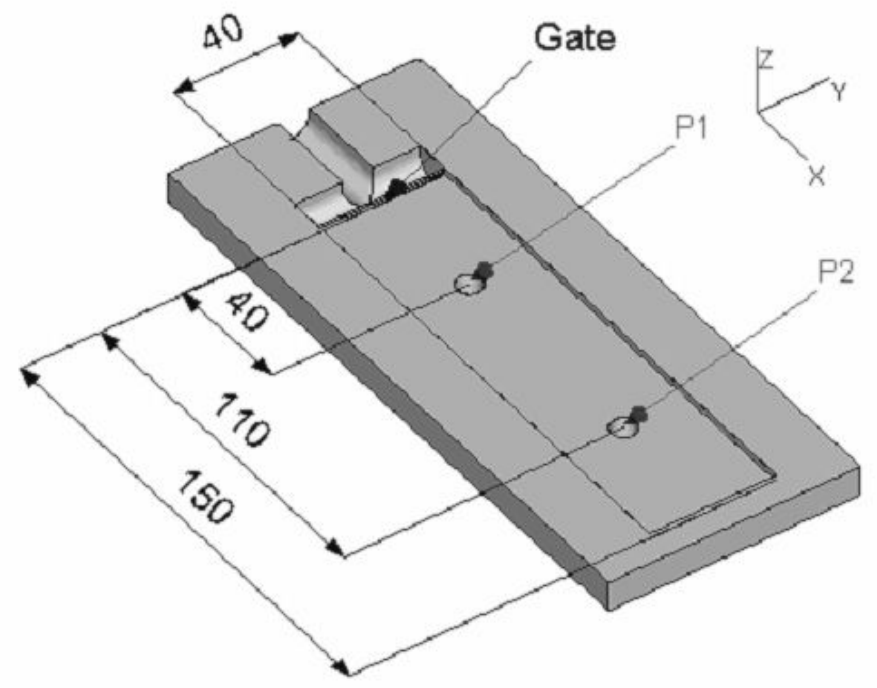

Fig. 2. Die insert of the injection molding part with $2 \mathrm{~mm}$ of thickness (dimensions in mm).

For the results produced by the modeling programme two process restrictions were imposed: i) the molding has to be completely filled, obviously no short-shots were admitted and ii) the computed values of the maximum shear stress and strain-rate were limited to their critical values (defined on the CMOLD database) in order to avoid potential defects (e.g., melt fracture).

The criteria used are the following:

1) The temperature difference on the molding at the end of filling, $d T=$ $\left(T_{\max }-T_{\min }\right)$, was minimized to avoid part distortions and warpage due to different local cooling rates, $d T \in[0 ; 20]^{\circ} \mathrm{C}$;

2) The volumetric shrinkage of the moldings was minimized, $V S \in[0 ; 15]$ $\%$;

3) The maximum mold pressure was minimized, reducing the clamping force, $P_{\max } \in[1 ; 70] \mathrm{MPa}$;

4) The cycle time was minimized, increasing productivity, $t_{c} \in[30 ; 35] \mathrm{s}$;

5) The pressure work, defined as the integral of pressure, $P$, along the time, $t$ :

$$
P W=\int_{0}^{t_{c}} P(t) d t
$$

was to be minimized in order to diminish the residual stress, the energy consumption and to reduce the mechanical efforts supported by the equipment, $P W \in[0 ; 200]$ MPa.s. 
The RPSGAe was applied using the following parameters: 50 generations, crossover rate of 0.8 , mutation rate of 0.05 , internal and external populations with 100 individuals, limits of the clustering algorithm set at 0.2 and NRanks $=30$. These values resulted from a carefully analysis made in a previous paper [11]. Since the aim of this work is to study the applicability of the optimization methodology in the injection molding process taking into account the various criteria identified above, the 20 optimization runs identified in Table 1 were carried out. Runs 1 to 9 considered 2 criteria simultaneously, runs 10 to 19 considered 3 criteria and run 20 all the 5 criteria.

Table 1. Criteria used in each process optimization run.

\begin{tabular}{lllll}
\hline Run & Optimized criteria & & Run & Optimized criteria \\
\cline { 1 - 2 } \cline { 5 - 5 } 2 & $V S$ and $d T$ & & 11 & $V S, d T$ and $t_{c}$ \\
2 & $V S$ and $P_{\max }$ & & 12 & $V S, d T$ and $P W$ \\
3 & $V S$ and $t_{c}$ & & 13 & $V S, P_{\max }$ and $t_{c}$ \\
4 & $V S$ and $P W$ & & 14 & $V S, P_{\max }$ and $P W$ \\
5 & $P W$ and $d T$ & & 15 & $V S, t_{c}$ and $P W$ \\
6 & $P W$ and $P_{\max }$ & & 16 & $P W, d T$ and $P_{\max }$ \\
7 & $P W$ and $t_{c}$ & & 17 & $P W, d T$ and $t_{c}$ \\
8 & $t_{c}$ and $d T$ & & 18 & $P W, P_{\max }$ and $t_{c}$ \\
9 & $t_{c}$ and $P_{\max }$ & & 19 & $t_{c}, d T$ and $P_{\max }$ \\
10 & $V S, d T$ and $P_{\max }$ & & 20 & All criteria \\
\hline
\end{tabular}

\section{Results and Discussion}

Fig. 3 shows the results obtained when 2 criteria are used simultaneously (for runs 4 to 7 as an example). The optimization algorithm is able to evolve during the 50 generations and to produce a good approximation to the Pareto frontier in all the cases.

For example, if run 4 is considered (VS - PW plot of Fig. 3), when solution 1 (VS $=3 \%$ and $P W=29.8 \mathrm{MPa} . \mathrm{s}$ ) is compared with solution 2 (VS $=1.1 \%$ and $P W$ = 199 MPa.s) in what concerns the operating conditions obtained, the most relevant changes are the injection temperature and the holding pressure (solution 1: $t_{i n j}$ $=2.6 \mathrm{~s}, T_{i n j}=207{ }^{\circ} \mathrm{C}, T_{w}=31{ }^{\circ} \mathrm{C}$ and $P h=7 \%$; solution $2: t_{i n j}=2.6 \mathrm{~s}, T_{i n j}=223$ ${ }^{\circ} \mathrm{C}, T_{w}=30{ }^{\circ} \mathrm{C}$ and $\left.P h=29 \%\right)$. Both changes contribute to the increase of $P W$ and consequently to the decrease of VS. The increment of $T_{i n j}$ decreases the melt viscosity, promoting a better packing of the material during the holding phase, so reducing shrinkage. Furthermore, increasing $P W$ does not increase the cycle time as would be expected. Interestingly, $P_{\max }$ and $d T$ both slightly decrease with $P W$, but the changes on these variables is small. 

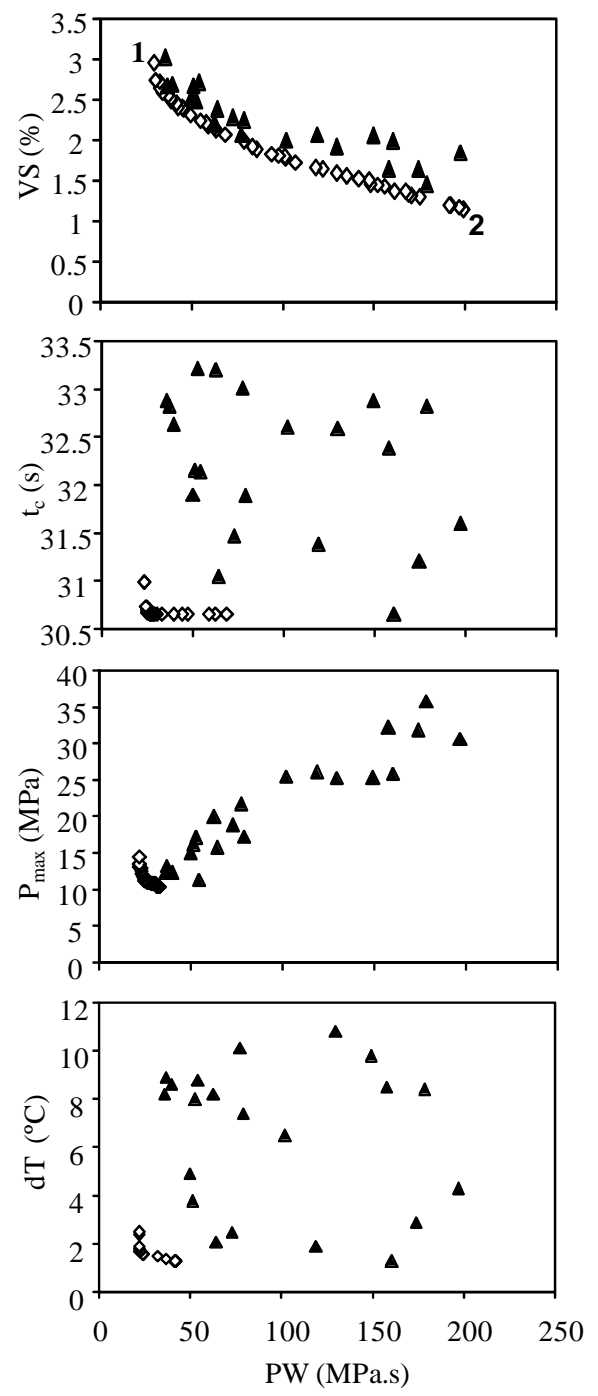

Fig. 3. Optimization results for two criteria, runs 4 to 7 . Open symbols: Pareto frontier at $50^{\text {th }}$ generation; full symbols: initial population ( $P W$ - Power Work, VS - volumetric shrinkage, $t_{c}-$ cycle time, $P_{\max }-$ maximum pressure, $d T$ - temperature difference).

Fig. 4 shows that when 3 criteria are used (Fig. 4 - left), a compromise between all the criteria considered is attained. Some of the Pareto solutions produced seem to be dominated in the individual 2 criteria plots. Identical conclusion can be made when 5 criteria are used (Fig. 5 - left): the Pareto frontier results from the compromise between all the 5 criteria. Figures 4 and 5 show also that the optimization 
algorithm is able to attain good solutions when compared with the 2 criteria cases (Figs. 4 and 5 - right).

Finally, the selection of a single solution (from the set of non-dominated solutions) to use in the real process depends on the articulation of preferences between the different criteria [16]. Due to its complexity, this is not subject of this text but will be considered in a near future [16].
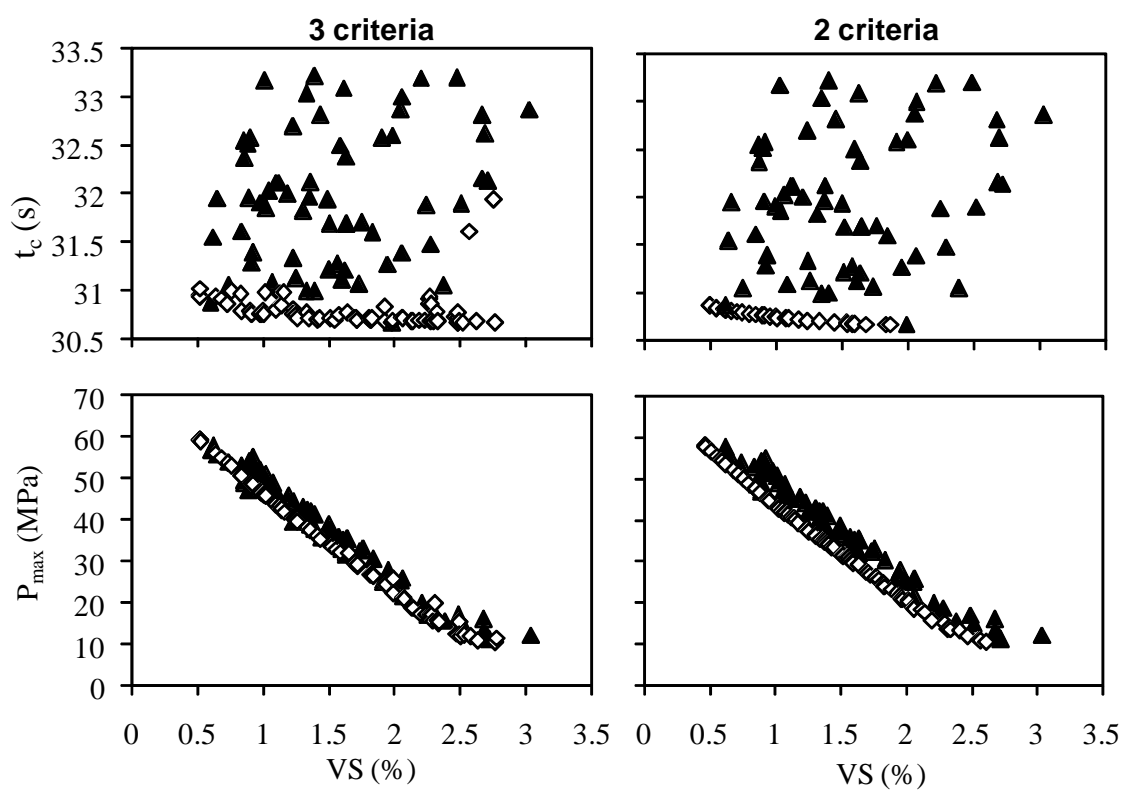

Fig. 4. Optimization results for 3 criteria of run 13 - left; and comparison with runs 2 and 3 right (open symbols: Pareto frontier at $50^{\text {th }}$ generation; full symbols: initial population).

\section{Conclusions}

A multi-objective optimization methodology based on Evolutionary Algorithms (MOEA) was applied in the optimization of the processing conditions of polymer injection molding process. The algorithm is able to take into account the multiple criteria used and, with a single run, to obtain a complete trade-off of solutions. The results produced have physical meaning which can be justified by the analysis of the process.

Acknowledgments. This work was supported by the Portuguese Fundação para a Ciência e Tecnologia under grant SFRH/BD/28479/2006. 

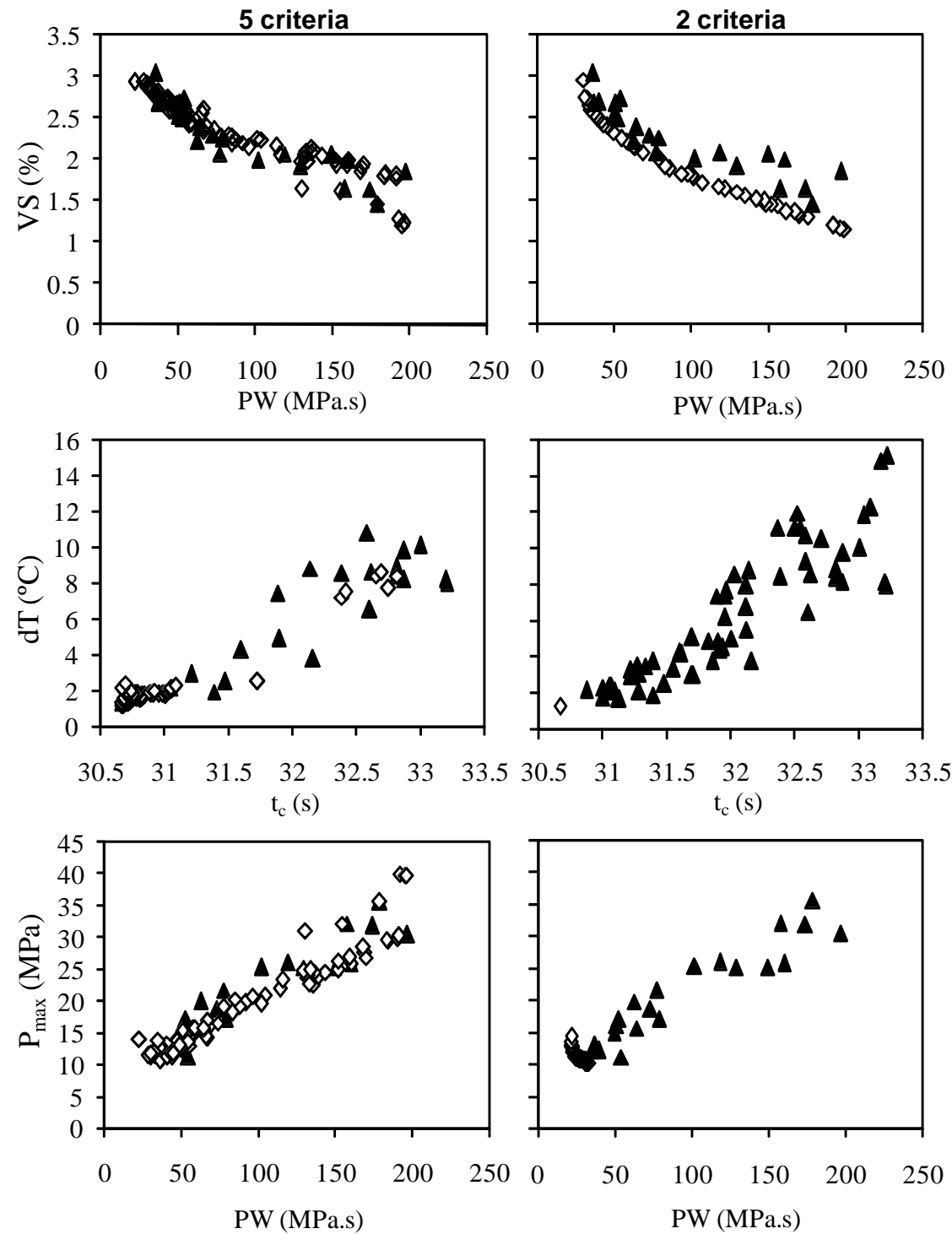

Fig. 5. Optimization results for 5 criteria of run 20 - left; and comparison with runs 4, 6 and 8 right (open symbols: Pareto frontier at $50^{\text {th }}$ generation; full symbols: initial population). 


\section{References}

1. Viana J C, Cunha A M, Billon N (2002) The thermomechanical environment and the microstructure of an injection moulded polypropylene copolymer. Polym 43:4185-4196

2. Viana J C (2004) Development of the skin layer in injection moulding: phenomenological model. Polym 45:993-1005

3. Cmold User's manual

4. Moldflow User's manual

5. Kim S J, Lee K, Kim Y I (1996) Optimization of injection-molding conditions using genetic algorithm. Proc of SPIE - the 4th Int Conf on Computer-Aided Design and Computer Graphics 2644:173-180

6. Lotti C, Bretas R E S (2003) The influence of morphological features on mechanical properties of injection molded PPS and sits prediction using neural networks. Proc of PPS 184-P

7. Castro C, Bhagavatula N, Cabrera-Rios M et al (2003) Identifying the best compromises between multiple performance measures in injection molding (IM) using data envelopment analysis. Proc SPE technical papers ANTEC'03

8. Turng L S, Peic M (2002) Computer aided process and design optimization for injection molding. J of Eng Manuf Proc of the Inst of Mech Eng (Part B) 12:1523-1532

9. Alam K, Kamal M R (2003) A genetic optimization of shrinkage by runner balancing. Proc SPE technical papers ANTEC'03 637-641

10.Cunha-Gaspar A, Viana J C (2005) Using multi-objective evolutionary algorithms to optimize mechanical properties of injection moulded parts. Int Polym Proc XX 3:274-285

11.Gaspar-Cunha A, Covas J A (2004) RPSGAe - reduced pareto set genetic algorithm: application to polymer extrusion. In: Gandibleux X, Sevaux M, Sörensen K et al (eds) Metaheuristics for multiobjective optimization. Springer-Verlag, Berlin Heidelberg

12.Goldberg D E (1989) Genetic algorithms in search, optimization and machine learning. Addison-Wesley, Boston

13.Gaspar-Cunha A (2000) Modelling and optimization of single screw extrusion. Ph D Thesis, University of Minho, Braga

14.Deb K (2001) Multi-objective optimization using evolutionary algorithms. Wiley, New York

15.Coello Coello C A, Van Veldhuizen D A, Lamont G B (2002) Evolutionary algorithms for solving multi-objective problems. Kluwer, Massachusetts

16.Ferreira J C, Fonseca C M, Gaspar-Cunha A (2007) Methodology to select solutions from the pareto-optimal set: a comparative study. GECCO 2007 Genetic and Evolutionary Computation Conference 789-796 